\title{
Children With Hearing Loss and Increased Risk of Injury
}

Josbua R. Mann, MD, MPH'

Li Zhou, MS ${ }^{2}$

Michael McKee, $M D^{3}$

Suzanne McDermott, $P b D^{1}$

'University of South Carolina School of Medicine, Department of Family and Preventive Medicine, Family Medicine Center, Columbia, SC

${ }^{2}$ University of South Carolina, Arnold School of Public Health, Department of Epidemiology and Biostatistics, Columbia, SC

${ }^{3}$ Lifetime Health, Folsom Family Center, Rochester, NY

\begin{abstract}
PURPOSE Few studies have tested the hypothesis that children with sensory disabilities such as deafness may be at increased risk of injuries. To test this hypothesis, this study compared rates of emergency department or hospital treatment for injury among Medicaid-insured South Carolina children with and without a diagnosis of hearing loss.
\end{abstract}

METHODS Medicaid billing data for 2002-2003 were obtained from the South Carolina Office of Research and Statistics. International Classification of Diseases, Ninth Revision, Clinical Modification billing codes were used to identify children with and without hearing loss, and episodes of injury-related emergency department or hospital treatment were compared for the 2 groups.

RESULTS Rates of injury treatment in children with hearing loss were more than twice that of the control group (17.72 vs 8.58 per 100, respectively). The relative rate $(R R)$ remained significantly higher $(R R=1.51,95 \%$ confidence interval, 1.30-1.75) after adjusting for age, race, sex, and the number of hospital or emergency department encounters for treatment of non-injury-related conditions. Children with hearing loss had significantly higher treatment rates for every injury type, bodily location, and external cause, with a cell size sufficient for valid comparison.

CONCLUSIONS Children with hearing loss may be at increased risk of injury. Additional study is needed to determine whether children with hearing loss are at increased risk (as opposed to simply seeking hospital care for injuries more often). If so, targeted injury prevention efforts for these children and their families would be warranted.

Ann Fam Med 2007;5:528-533. DOI: 10.1370/afm.740.

\section{INTRODUCTION}

Thury is the leading cause of death in children in the United States, accounting for more than 14,000 deaths in children aged 18 years or 1 younger in $2004 .^{1}$ It is estimated that more than 18 million nonfatal injuries occur annually in Americans younger than 20 years, with 13 million of these occurring in children younger than 15 years. ${ }^{2}$ The estimated annual cost for medical care owing to injuries in children is approximately $\$ 17$ billion; when estimated costs resulting from lost future work and loss in quality of life are included, the cost increases to more than $\$ 300$ billion. $^{2}$

It has been hypothesized that children with sensory disabilities (blindness or deafness) may be at increased risk of injuries as a result of difficulties identifying and responding to hazards in the environment. ${ }^{3}$ Although the prevalence of moderate to profound, bilateral, permanent hearing loss in newborns in industrialized nations is between 1 in 900 and 1 in 2,500, ${ }^{4}$ at least some hearing loss may be found in as many as $11 \%$ of school-aged American children. ${ }^{5}$ There is a general lack of surveillance and research regarding injuries in children with disabilities, ${ }^{5}$ and we were able to identify only 1 article about the risk of injury in children with hearing loss. Roberts and Norton studied the risk of pedestrian-motor vehicle collisions 
in children in New Zealand and found that the odds of death or hospitalization caused by such an injury was approximately twice as high in children with hearing loss. ${ }^{6}$ Research on occupational injuries in adults with hearing loss has shown an increased risk of approximately the same magnitude., ${ }^{7,8}$

This study is a first effort to describe the rates of emergency department and hospital treatment for injury in American children with hearing loss and compare those rates with the rates for a control group with no hearing loss.

\section{METHODS}

We measured injury frequency for 138,111 South Carolina children aged 1 through 18 years who were insured by Medicaid throughout the 12 months of 2003. The data included both eligibility and claims data obtained from the South Carolina Office of Research and Statistics. We also obtained 2002 billing data for all children included in the 2003 file.

Our research was broadly focused on children with a number of disabilities: mental retardation, developmental delay, autism, spinal cord injury, cerebral palsy, hearing loss, and vision loss. We provided the South Carolina Office of Research and Statistics (ORS) with a list of International Classification of Diseases, Ninth Revision, Clinical Modification (ICD-9-CM) codes for these conditions. They then provided us with a file that included all the children with 1 of these diagnoses in 2003, plus an age-matched file of comparison children at a 2-to-1 ratio. A linked file for 2002 was also provided. Records were obtained for approximately 46,000 children with 1 of these diagnoses, in addition to 92,000 children without 1 of the disabilities listed above. The data included demographic information (age, race, and sex), monthly eligibility information, diagnosis code and dates of admission to the hospital or emergency department, and E codes for external causes of injury. For each record, there is 1 field for primary diagnosis and 8 fields for secondary diagnoses; all 9 fields were used in identifying the children with hearing loss, as well as in defining the treatment of injuries.

The identification of children with hearing loss was based on an ICD-9-CM code for hearing loss. These codes are 389.0 through 389.9 . Of the 46,000 children with 1 disability listed above, 4,647 had a diagnosis of hearing loss in 2003. We further modified the hearing loss group by requiring that the hearing loss diagnosis be found in both 2002 and 2003 for 2 reasons: first, it increases the probability that the diagnosed hearing loss was long-term rather than transient, second, it permits prospective data analysis, because the hearing loss diagnosis had to be present in 2002, before the occurrence of injury treatment in 2003. Children with a diagnosis of hearing loss only in 2002 or 2003 were excluded from both the hearing loss and comparison groups.

To reduce confounding from coexisting disabilities, children were excluded from both the hearing loss and comparison groups if in 2003 they had an ICD-9-CM diagnosis of mental retardation, autism, cerebral palsy, or vision loss. Children with diagnosed developmental delay were excluded, except for children with developmental speech or language disorder (ICD-9-CM code 315.3) because delays in speech can result from hearing loss. Children with diagnosed spinal cord injuries in 2002 were excluded, but spinal cord injury in 2003 was one of the outcomes of interest. After these exclusions, there were 1,010 children in the hearing loss group and 91,591 in the comparison group.

We focused the analysis on the number of injuryrelated episodes of emergency department or hospital care for children with and without hearing loss. Each episode of care was defined as an emergency department visit or hospitalization, with an ICD-9-CM code indicative of injury, on a given day. Hospital treatment on consecutive days was considered a single episode of care. If a child received emergency department and hospital care on the same day, the visits were regarded to be only 1 episode of care.

We counted the total number of injury-related episodes of care for each child. The total number of injury treatment episodes for any cause was counted as 1 outcome. For this overall rate, only 1 count per episode of care was included. For example, if a child was treated for a leg fracture and a traumatic brain injury on the same day, they were counted as only 1 episode of injury in the overall rate.

We also counted the number of episodes for each specific type (eg, fracture, dislocation, strain/sprain) or location (eg, upper extremity, lower extremity, face) of injury, according to the Barell Injury Matrix, ${ }^{9}$ which has 432 detailed injury categories. For simplicity, however, we collapsed the categories into 8 location groupings and 7 injury types. Each location or type of injury was counted independently, so that a count was given for a specific type or location of injury if the ICD-9-CM codes for that type or location of injury were found in an episode of care. For this outcome, the hypothetical child who experienced both a leg fracture and a traumatic brain injury on the same day would be counted in the outcome group for both leg fracture and traumatic brain injury. We also used E codes to evaluate the nature of the incident that caused the injury (eg, fall, poisoning). E codes were categorized based on the most recent National Vital Statistics report on mortality that used ICD-9-CM E codes for reporting injury deaths. ${ }^{10}$ 
We modeled the number of emergency department or hospital encounters for injury using Poisson regression, adjusting for age, race, and sex. Poisson regression was selected because it is the most appropriate statistical method when the dependent variable is measured as a number of occurrences over a specific time. Treatment for any injury was modeled, as were specific injuries as identified by injury type, bodily location, and external cause. Each emergency department or hospital encounter for injury was treated as a separate occurrence. To address the possibility that a few outliers could account for inordinate numbers of encounters, we also modeled, using logistic regression, the odds of at least 1 emergency department or hospital encounter caused by injury. The results were not substantially different, so we elected to report the Poisson regression results because that approach is the most straightforward.

\section{RESULTS}

Descriptive statistics for children with and without hearing loss are displayed in Table 1. Children with hearing loss were significantly more likely to be white and male. They were also more likely to be at the younger or older end of the age range and less likely to be from 5 to 12 years old.

The distribution of hearing loss ICD-9-CM codes is displayed in Table 2. Almost three-fourths of the children had only 1 code for hearing loss; most of whom ( $41 \%$ of the total) had sensorineural hearing loss or combined conductive and sensorineural hearing loss diagnosed (ICD-9-CM 389.1 or 389.2 ). Of the $26 \%$ of children with more than 1 hearing loss code assigned at some point in 2002 and 2003, a great majority had at least 1 code indicative of sensorineural hearing loss. Thus, almost two-thirds of the 1,010 children with loss of hearing had at least some component of sensorineural hearing loss. This finding is consistent with our efforts to limit our analyses as much as possible to children with serious, long-term hearing loss, which is most often sensorineural. ${ }^{11,12}$

The rates of overall injury treatment and treatment for specific categories of injury in children who have hearing loss and comparison children are displayed in Table 3 . The overall rate of emergency department or hospital treatment for injury was 17.72 per 100 episodes for children with hearing loss and 8.58 per 100 episodes for comparison children. The probability of experiencing at least 1 episode of injury was similarly elevated: $15.4 \%$ vs $7.1 \%$. In the Poisson regression model predicting overall injury treatment rates, male sex, older age, and white race were all significant predictors of increased injury treatment rates. Children with hearing loss had significantly higher injury treatment rates after controlling for these demographic variables (relative rate $[\mathrm{RR}]=1.98 ; 95 \%$ confidence interval [CI], 1.71-2.30) (Table 4).

Regression analyses predicting specific types of injury were conducted only on categories with at least 10 observations for each group. The results are displayed in Table 4. For each injury category with enough counts to be adequate for modeling, children with hearing loss experienced significantly higher rates of injury treatment. In each case, the relative rate was around 2.0, and each relative rate was highly significant $(P<.01)$.

Table 1. Demographic Characteristics of Children With Hearing Loss and Comparison Group

\begin{tabular}{lcccc}
\hline & \multicolumn{2}{c}{$\begin{array}{c}\text { Hearing } \\
\text { Loss Group }\end{array}$} & \multicolumn{2}{c}{$\begin{array}{c}\text { Comparison } \\
\text { Group }\end{array}$} \\
\cline { 2 - 5 } Characteristics & No. & $\%$ & No. & $\%$ \\
\hline Age* $^{*}$ 1-4 years & 190 & 18.8 & 14,762 & 16.1 \\
5-12 years & 552 & 54.7 & 58,007 & 63.3 \\
13-18 years & 268 & 26.5 & 18,822 & 20.6 \\
Sex & & & & \\
Male & 541 & 53.6 & 45,918 & 50.1 \\
Female & 469 & 46.4 & 45,673 & 49.9 \\
Race* & & & & \\
African American & 415 & 41.1 & 48,309 & 52.8 \\
White & 510 & 50.5 & 37,900 & 41.4 \\
Other & 85 & 8.4 & 5,382 & 5.9 \\
\hline * $P=<.001$. & & & & \\
$\dagger P=<.05$. & & & & \\
\hline
\end{tabular}

Table 2. ICD-9-CM Codes for Hearing Loss and Children With Diagnosis

\begin{tabular}{llc}
\hline Number of Codes & ICD-9-CM Code & $\begin{array}{c}\text { Children } \\
\text { With Code } \\
\text { No. (\%) }\end{array}$ \\
\hline 1 code $(\mathrm{n}=747,74.0 \%)$ & $\begin{array}{l}389.0 \text { (conductive hearing loss) } \\
389.1 \text { (sensorineural hearing loss) }\end{array}$ & $\begin{array}{c}243(24.1) \\
393(38.9)\end{array}$ \\
& $\begin{array}{c}389.2 \text { (mixed conductive and } \\
\text { sensorineural hearing loss) }\end{array}$ & $15(1.5)$ \\
& 389.9 (unspecified hearing loss) & $96(9.5)$ \\
2 codes $(n=211,20.9 \%)$ & 389.1 \& 389.9 & $101(10.0)$ \\
& 389.0 \& 389.1 & $41(4.1)$ \\
& 389.0 \& 389.9 & $30(3.0)$ \\
3 or more codes & Other 2-code combinations & $39(3.9)$ \\
$(\mathrm{n}=52,5.1 \%)$ & $389.0,389.1$, and 389.9 & $33(3.3)$ \\
Other combinations of & & $19(1.9)$ \\
3 or more codes & & \\
\hline ICD-9-CM = International Classification of Disease, Ninth Edition, Clinical Modification.
\end{tabular}


Table 3. Injury Rates in Children by Hearing Loss Status

\begin{tabular}{lcccc}
\hline & \multicolumn{2}{c}{ Hearing Loss Group } & \multicolumn{2}{c}{ Comparison Group } \\
\cline { 2 - 5 } Injury & No. & Rate per 100 & No. & Rate per 100 \\
\hline Overall rate & 179 & 17.72 & 7,863 & 8.58 \\
Traumatic brain injury & 1 & 0.10 & 87 & 0.09 \\
Other head, face, and neck & 49 & 4.85 & 2,125 & 2.32 \\
Spinal cord injury & 0 & 0.00 & 1 & 0.00 \\
Vertebral column injury & 2 & 0.20 & 278 & 0.30 \\
Torso & 9 & 0.89 & 510 & 0.56 \\
Upper extremity & 73 & 7.23 & 2,660 & 2.90 \\
Lower extremity & 50 & 4.95 & 2,214 & 2.42 \\
Other and unspecified & 5 & 0.50 & 372 & 0.41 \\
Fracture & 26 & 2.57 & 1,054 & 1.15 \\
Dislocation & 3 & 0.30 & 137 & 0.15 \\
Sprains and strains & 29 & 2.87 & 1,394 & 1.52 \\
Internal & 1 & 0.10 & 98 & 0.11 \\
Oppen wound & 40 & 3.96 & 1,807 & 1.97 \\
Contusion, superficial & 72 & 7.13 & 2,738 & 2.99 \\
Burns & 5 & 0.50 & 275 & 0.30 \\
\hline
\end{tabular}

emergency department or hospital care at a markedly elevated rate $(\mathrm{RR}=4.26$; $95 \% \mathrm{CI}, 4.07-4.46)$. We reran the model predicting injury treatment, controlling for the number of non-injury-related emergency department or hospital encounters in addition to age, race, and sex. After controlling for non-injuryrelated emergency department or hospital utilization, children with hearing loss still had increased rates of injury treatment $(\mathrm{RR}=1.51 ; 95 \% \mathrm{CI}, 1.30-1.75)$.

The ICD-9-CM coder's manual ${ }^{13}$ provides categories for "suicide and self-inflicted injury" and "homicide and injury purposely inflicted by other persons." There were no children with hearing loss with intentional self-injury diagnosed and only 2 (0.002 per child) had intentional injury by others diag-
Table 4. Poisson Regression, Adjusted Relative Rate of Injury Site and Type

\begin{tabular}{lcccc}
\hline Injury Category & RR & $\mathbf{9 5 \%} \mathbf{C l}$ & $\chi^{2}$ & $P$ Value \\
\hline Any injury & 1.98 & $1.71-2.30$ & 82.13 & $<.001$ \\
Other head, face, & & & & \\
$\quad$ and neck & 2.04 & $1.53-2.70$ & 24.15 & $<.001$ \\
Upper extremity & 2.28 & $1.80-2.87$ & 47.99 & $<.001$ \\
Lower extremity & 1.88 & $1.42-2.49$ & 19.54 & $<.001$ \\
Fracture & 2.02 & $1.37-2.99$ & 12.61 & $<.001$ \\
Sprains and strains & 1.64 & $1.14-2.38$ & 7.02 & .008 \\
Open wound & 1.92 & $1.41-2.63$ & 16.75 & $<.001$ \\
Contusion, superficial & 2.27 & $1.80-2.87$ & 47.20 & $<.001$ \\
\hline
\end{tabular}

Note: Sex, age, and race are included as covariates in each model, and relative rates are for children with hearing loss compared to controls. Only outcomes with at least 10 occurrences in the hearing loss and comparison group are included.

$\mathrm{RR}=$ relative rate $\mathrm{Cl}=$ confidence interval.

Rates of injury by external cause (E codes) are shown in Table 5 . E code data were available for $77 \%$ of encounters both with and without hearing loss. Only 4 categories of E codes had adequate counts in both groups to allow Poisson regression modeling: cut or pierce, fall, struck by or against, other specified and unspecified. For each of these specific categories of injury causation, the rate of treatment was significantly higher in children with hearing loss.

We were concerned that the elevated rates of injury treatment among children with hearing loss could be due to an increased propensity to pursue emergency or hospital treatment. To address these concerns, we calculated rates of emergency department or hospital treatment for any reason other than injury. Adjusting for age, race, and sex, children with hearing loss used nosed. Nineteen comparison children $(0.0002$ per child) had self-injury diagnosed, and 145 (0.0016 per child) had intentional injury by others diagnosed. Poisson modeling was not performed for these outcomes because of the small cell sizes in the hearing loss group.

\section{DISCUSSION}

Our analyses show that South Carolina children with hearing loss, insured by Medicaid, receive emergency department or hospital treatment for injuries at a significantly higher rate than do children without a disability. The association was found for most types of injuries, most bodily locations, and most external causes, and it persists when controlling for emergency department or hospital visits for reasons other than injury. Thus, it appears to be quite robust.

The most important weakness of this study is its reliance on billing records, which allows us to comment only on the rates of injury treatment, not the actual rates of injury. Differences in the propensity of parents to pursue treatment for children's injuries could influence the findings. Another weakness of using billing data is that these data are based on physician billing codes for outcome ascertainment and for the identification of children with hearing loss. Iezzoni ${ }^{14}$ discusses at length the problems with using billing records to identify people with disabilities. Two primary problems apply to our study: (1) some children who have hearing loss were likely not identified in the data, and (2) we do not have an objective measure of degree of hearing loss, so our analyses have necessarily lumped together children with a wide range of hearing impairment. 


\begin{tabular}{|c|c|c|c|c|c|c|}
\hline \multirow[b]{2}{*}{ External Cause } & \multicolumn{2}{|c|}{$\begin{array}{l}\text { Hearing } \\
\text { Loss Group }\end{array}$} & \multicolumn{2}{|c|}{$\begin{array}{c}\text { Comparison } \\
\text { Group }\end{array}$} & \multicolumn{2}{|c|}{$\begin{array}{l}\text { Hearing Loss vs } \\
\text { Comparison Group }\end{array}$} \\
\hline & No. & $\begin{array}{c}\text { Rate } \\
\text { per } 100\end{array}$ & No. & $\begin{array}{c}\text { Rate } \\
\text { per } 100\end{array}$ & $\begin{array}{c}\text { Adjusted } \\
\text { Rate Ratio* }\end{array}$ & $95 \% \mathrm{Cl}$ \\
\hline Fall & 48 & 4.75 & 1,531 & 1.67 & 2.75 & $2.06-0.67$ \\
\hline Other specified & 32 & 3.17 & 1,378 & 1.50 & 2.02 & $1.42-2.87$ \\
\hline Struck by or against & 23 & 2.28 & 1,243 & 1.36 & 1.58 & $1.05-2.39$ \\
\hline Cut or pierce & 11 & 1.09 & 498 & 0.54 & 1.89 & $1.04-3.43$ \\
\hline $\begin{array}{l}\text { Motor vehicle } \\
\text { traffic, all }\end{array}$ & 6 & 0.59 & 460 & 0.50 & & \\
\hline $\begin{array}{l}\text { Natural or } \\
\text { environmental }\end{array}$ & 6 & 0.59 & 402 & 0.44 & & \\
\hline Pedal cyclist, other & 5 & 0.50 & 242 & 0.26 & & \\
\hline $\begin{array}{l}\text { Fire or hot object } \\
\text { or substance }\end{array}$ & 3 & 0.30 & 93 & 0.10 & & \\
\hline Poisoning & & & 85 & 0.09 & & \\
\hline $\begin{array}{l}\text { Transportation, } \\
\text { all other }\end{array}$ & 3 & 0.30 & 83 & 0.09 & & \\
\hline Firearm & & & 35 & 0.04 & & \\
\hline Suffocation & & & 9 & 0.01 & & \\
\hline Machinery & & & 4 & 0.004 & & \\
\hline Pedestrian, other & 1 & 0.10 & 4 & 0.004 & & \\
\hline Drowning & & & 3 & 0.003 & & \\
\hline
\end{tabular}

and assets are considered. We obtained information on whether the children in this analysis had qualified for Medicaid on the basis of low income or disability. Two percent of children in the comparison group qualified because of a disability, compared with $29 \%$ of those with hearing loss. Thus, it appears that comparison children were more likely to be from low-income families. If low income is associated with a greater likelihood of emergency department or hospital injury treatment, the actual effect of hearing loss may actually be greater than reported in this article.

Another potential confounder is that children with hearing loss may be at higher risk of other medical conditions or disabilities. For example, 30\% of newborns with substantial hearing loss also have neurological disorders, ${ }^{8}$ and

E codes are also imperfect for assessing cause of injury, since they are open to the physician's understanding of the situation, which may be incomplete. In addition, physicians may be reluctant to assign an E code indicative of intentional injury or abuse. A recent survey of pediatricians in Pennsylvania found wide variation in their opinions of what would constitute reasonable suspicion of child abuse. ${ }^{15}$ Thirty-five percent of those surveyed reported that for reasonable suspicion to exist, the probability of abuse needed to be $10 \%$ to $35 \%$. Contrarily, $40 \%$ reported that the probability of abuse would need to be $60 \%$ or more to represent reasonable suspicion of child abuse. The low incidence of $\mathrm{E}$ codes indicating intentional injury by others in both the hearing loss and comparison groups may represent underreporting by physicians.

Children with hearing loss are more likely to be poor, white, and male. ${ }^{16}$ One might suspect the relationship between hearing loss and injury to be confounded by demographics and socioeconomic status. We report rate ratios that are adjusted for age, sex, and race. We did not, however, have data on socioeconomic status. Although most children covered by Medicaid in South Carolina have family incomes of less than $150 \%$ of the poverty level, children with disabilities who need institutional level care can qualify for Medicaid coverage without regard to the family's income or assets-only the child's personal income almost $40 \%$ have some comorbidity. ${ }^{9}$ For this study, children with any codiagnosis of mental retardation, learning disability, autism, cerebral palsy, spinal cord injury, or vision loss were excluded. There were, however, some children likely to have these potential confounders who were not identified in the billing data. There may also have been other conditions that were more common among children with hearing loss and that accounted for some of the observed increase in injury treatment rates.

All these caveats aside, it is reasonable to believe that children with hearing loss may be at higher risk of injury. The most straightforward explanation may be reduced ability to recognize danger clues. In addition to difficulty hearing the noise associated with hazards (eg, motorized vehicles, footsteps), children with hearing loss also may have difficulty hearing a parent's warning of impending danger. Further, because most children who are deaf or hard of hearing have parents without hearing loss, there may be underlying deficiencies in anticipatory education about hazards if parent-child communication is suboptimal. Additional research would be needed to test this hypothesis.

In summary, South Carolina children with hearing loss, insured by Medicaid, receive emergency department and hospital care for injuries at higher rates than children with no disability. Further study using direct measurement of hearing loss and of injury outcomes 
is needed. If additional research verifies that children with hearing loss are indeed at higher risk of injury, efforts to develop injury prevention interventions designed specifically for these children and their families would seem to be warranted. Meanwhile, clinicians should consider discussing injury prevention strategies with parents of children with hearing loss. A discussion of the implications of a child's diminished ability to hear verbal or nonverbal warnings could be a central aspect of this counseling.

To read or post commentaries in response to this article, see it online at http://www.annfammed.org/cgi/content/full/5/6/528.

Key words: Hearing impaired persons; hearing loss; wounds and injuries

Submitted December 5, 2006; submitted, revised, May 21, 2006; accepted May 24, 2006.

Funding support: The research in this publication was funded by the Centers for Disease Control Health and Disability Cooperative Agreement U59/CCU421834.

\section{References}

1. National Center for Injury Prevention and Control. WISQARS leading causes of death reports, 2004. http://webappa.cdc.gov/sasweb/ ncipc/leadcaus10.html. Accessed April 11, 2007

2. Danseco ER, Miller TR, Spier RS. Incidence and costs of 1987 1994 childhood injuries: demographic breakdowns. Pediatrics. 2000;105(2):e27.

3. Gaebler-Spira D, Thornton LS. Injury prevention for children with disabilities. Phys Med Rehabil Clin N Am. 2002;13(4):891-906.
4. Thompson DC, McPhillips H, Davis RL, Lieu TA, Homer CJ, Helfand $M$. Universal newborn hearing screening: summary of evidence. JAMA. 2001;286(16):2000-2010.

5. Bess FH, Dodd-Murphy J, Parker RA. Children with minimal sensorineural hearing loss: prevalence, educational performance, and functional status. Ear Hear. 1998;19(5):339-354.

6. Roberts I, Norton R. Sensory deficit and the risk of pedestrian injury. Inj Prev. 1995;1(1):12-14.

7. Zwerling C, Sprince NL, Davis CS, Whitten PS, Wallace RR, Heeringa SG. Occupational injuries among older workers with disabilities: a prospective cohort study of the Health and Retirement Survey, 1992 to 1994. Am J Public Health. 1998;88(11):1691-1695.

8. Choi SW, Peek-Asa C, Sprince N, et al. Hearing loss as a risk factor for agricultural injuries. Am J Ind Med. 2005;48(4):293-301.

9. Barell V, Aharonson-Daniel L, Fingerhut LA, et al. An introduction to the Barell body region by nature of injury diagnosis matrix. Inj Prev. 2002;8(2):91-96.

10. Murphy SL. Deaths: Final Data for 1998. National Vital Statistics Reports. Hyattsville, MD: National Center for Health Statistics; 2000:48(11).

11. Van Naarden K, Decoufle P, Caldwell K. Prevalence and characteristics of children with serious hearing impairment in metropolitan Atlanta, 1991-1993. Pediatrics. 1999;103(3):570-575.

12. Davidson J, Hyde ML, Alberti PW. Epidemiology of hearing impairment in childhood. Scand Audiol Suppl. 1988;30:13-20.

13. Hart AC, Hopkins CA. ICD-9-CM Professional for Physicians, Volumes 1 \& 2. 6th ed. St. Anthony Publishing. Reston, VA; 2001.

14. Iezzoni LI. Using administrative data to study persons with disabilities. Milbank Q. 2002;80(2):347-379.

15. Levi BH, Brown G. Reasonable suspicion: a study of Pennsylvania pediatricians regarding child abuse. Pediatrics. 2005;116(1):e5-e12.

16. Holt J, Hotto S, Cole K. Demographic Aspects of Hearing Impairment Questions and Answers. 3rd Ed. Washington, DC: GRI Galludet University; 1994. 